\title{
Factors Governing the Solubilization of Phosphopeptides Retained on Ferric NTA IMAC Beads and Their Analysis by MALDI TOFMS
}

\author{
S. R. Hart, ${ }^{*}$ M. D. Waterfield, ${ }^{*}$ A. L. Burlingame, ${ }^{*,+}$ and R. Cramer* \\ Ludwig Institute for Cancer Research, London, United Kingdom
}

We have revisited the direct analysis experiments reported by Tomer and co-workers in the MALDI-TOFMS analysis of phosphopeptide-loaded immobilized metal ion affinity chromatography (IMAC) beads (Zhou, W.; Merrick, B. A.; Khaledi, M. G.; Tomer, K. B. J. Am. Soc. Mass Spectrom. 2000, 11, 273-282). The results described herein provide no evidence to support a laser-induced direct desorption of phosphopeptides chelated on IMAC beads. However, we have established that solubilization of mono-phosphopeptides from their immobilized $\mathrm{Fe}^{3+}$ NTA chelates does occur effectively in solutions containing certain MALDI matrices. Particularly effective is 2,5-dihydroxybenzoic acid (2,5-DHB), which apparently forms a stronger chelation complex with $\mathrm{Fe}^{3+}$-NTA than mono-phosphopeptides. With regard to the disparity observed between the low $\mathrm{pH}$ value of MALDI matrices (saturated 2,5-DHB $\mathrm{aq}_{\mathrm{ar}} \sim \mathrm{pH}$ ) and the high $\mathrm{pH}$ values of conventional IMAC eluents (typically above $\mathrm{pH} 7$ ), we have also investigated the influence of eluent $\mathrm{pH}$ on the recovery of phosphopeptides from IMAC media. Finally, we have confirmed the importance of employing ammonium dihydrogen phosphate as buffer to achieve effective liberation of mono- and all poly-phosphopeptide species from $\mathrm{Fe}^{3+}$-NTA IMAC resin. (J Am Soc Mass Spectrom 2002, 13, 1042-1051) (C) 2002 American Society for Mass Spectrometry

$\mathrm{I}$ mmobilized metal ion affinity chromatography (IMAC) has been used for a number of years for the selective enrichment of phosphopeptides from proteolytic digest mixtures containing both phosphorylated and non-phosphorylated components [1-8]. The established methods have largely relied upon the use of high-pH elution buffers to disrupt the interaction of phosphopeptides remaining bound to the metal ioncontaining IMAC media after separation of all other peptides [2-5].

In addition to the usual conditions documented above for solubilization of peptides bound in their immobilized, chelated form attached to the resin, evidence has been presented which suggests that monophosphopeptides may be released directly from the resin during the MALDI process [7,9]. It was recognized that the ferric IMAC resin must bind multiple phosphorylated components as well, but that laser irradia-

Published online July 24, 2002

Address reprint requests to Dr. R. Cramer, Ludwig Institute for Cancer Research, Cruciform Building, Gower Street, London WC1E 6BT, UK. E-mail: rainer@ludwig.ucl.ac.uk

*Also at Department of Biochemistry and Molecular Biology, University College London, Gower Street, London WC1E 6BT, UK.

${ }^{+}$Also at Department of Pharmaceutical Chemistry, University of California, 513 Parnassus Ave., San Francisco, CA 94143-0446, USA. tion does not easily dissociate these components from the agarose IMAC beads [7].

While it was suggested that laser desorption directly from IMAC beads occurs particularly for mono-phosphorylated peptide components, in these reports it was also questioned whether or not the IMAC analytes are still bound to the beads through their affinity interaction immediately prior to laser desorption $[9,10]$. Therefore, the possibility exists that "elution" from the beads into the MALDI matrix solution/crystals has in fact taken place prior to MALDI desorption and mass analysis.

Of course, there would be clear advantages in having a protocol that permits the direct analysis of phosphopeptides from IMAC beads, particularly if the analyte species thus detected could be qualitatively and quantitatively representative of the components bound to the resin. However, if phosphopeptide desorption takes place directly from their chelated form on the beads, probably the majority of bound phosphopeptides would not be accessible for laser desorption because of the unavoidable "shadow effect" of the threedimensional resin beads. On the other hand, if the matrix itself were able to "solubilize" the bound phosphopeptides, then further detailed investigations would be desirable in order to understand the potential analytical utility of such a process. 
To this end, we have chosen to reinvestigate the nature of the "direct analysis" phenomenon and wish to report that the acidic MALDI matrix, 2,5-dihydroxybenzoic acid (2,5-DHB), is able to liberate chelated monophosphopeptides from $\mathrm{Fe}^{3+}$-NTA resin because of its apparently higher chelation affinity for immobilized $\mathrm{Fe}^{3+}$ ions. In addition, we have established that multiply phosphorylated peptides remain bound under these conditions and in fact require the use of an ammonium dihydrogen phosphate buffer to effect disruption and solubilization from the ferric NTA resin, although not necessarily under the alkaline buffer conditions commonly employed as discussed above. As a consequence, we have systematically investigated the reaction conditions required to assure the liberation of phosphopeptides of varying degrees of modification from IMAC resins.

\section{Experimental}

Nickel loaded nitrilotriacetic acid (NTA) beads from Qiagen (Hilden, Germany) were used throughout our studies, since it has been shown by a number of research groups that NTA is a more effective chelating agent for use in IMAC than the more widely used iminodiacetic acid. This effectiveness appears to be due to its tetradentate structure and therefore stronger bead-metal ion interaction [7, 11]. The Ni-NTA beads were washed in three volumes of water to remove their antibacterial $50 \%$ aqueous ethanol storage solution. Bound divalent $\mathrm{Ni}^{2+}$ ions were removed by chelation with EDTA (three volumes of $100 \mathrm{mM}$ solution) with subsequent rinsing in water. Next, the $\mathrm{pH}$ was adjusted to 2.5 by rinsing with $100 \mathrm{mM}$ acetic acid solution, and the iron-loading step was performed with $100 \mathrm{mM}$ $\mathrm{FeCl}_{3}$ dissolved in $100 \mathrm{mM}$ acetic acid. The beads were then washed in $100 \mathrm{mM}$ acetic acid and stored at $4^{\circ} \mathrm{C}$. Beads are chemically stable under these storage conditions for a period of several weeks, so bulk volumes can be prepared in advance.

HPLC grade water was purchased from Rathburn (Walkerburn, UK), modified porcine trypsin from Promega (Madison, WI), and all other chemicals from Sigma-Aldrich (Poole, UK). MALDI-TOFMS mass spectra were recorded on either a Reflex III (Bruker Daltonik, Bremen, Germany) or a Voyager Elite XL (Applied Biosystems, Foster City, CA) reflectron time-offlight mass spectrometer.

Matrix solutions were prepared as follows: 2,5-DHB (Bruker Daltonik), was prepared as a saturated aqueous solution in HPLC grade water. CHCA was either used as a ready-made solution in methanol (Hewlett-Packard, Palo Alto), or made up at $10 \mathrm{mg} / \mathrm{ml}$ in $45 \%$ ethanol, $10 \%$ formic acid.

For the present study, we have essentially employed the method described by Zhou et al. [7]. Briefly, $10 \mu \mathrm{l}$ of settled $\mathrm{Fe}^{3+}$-charged IMAC resin was mixed with a tryptic digest of the commercially available (SigmaAldrich, Poole, UK), hyper-phosphorylated protein $\alpha$-casein $(10 \mu \mathrm{l}$ of a $2 \mathrm{pmol} / \mu \mathrm{l}$ solution in $100 \mathrm{mM}$ acetic acid) for $15 \mathrm{~min}$ on a rotator at room temperature. The supernatant was discarded and any non-specifically adsorbed peptides were washed off using $3 \times 20 \mu \mathrm{l}$ washes in each of $100 \mathrm{mM}$ acetic acid and HPLC grade water. Affinity-bound phosphopeptides were analyzed directly from the beads by loading $0.3-0.5 \mu l$ of the bead slurry to a MALDI target, adding 0.5-1 $\mu$ l of matrix solution and drying the sample under a warm stream of air (direct analysis). Alternatively, phosphopeptides were displaced from IMAC beads offtarget by employing the same matrix solutions as used for direct analysis. In this case, following treatment with matrix solution, the "eluted" supernatant was applied to the MALDI target and dried without additional matrix (indirect analysis). In the third set of experiments, in which the $\mathrm{pH}$ of the elution buffer was varied, phosphopeptides were eluted with $100 \mathrm{mM}$ ammonium phosphate buffer. This buffer was adjusted to various $\mathrm{pH}$ values using ammonium hydroxide. In this case, the MALDI sample was prepared by spotting $0.5 \mu \mathrm{l}$ of the eluent on the MALDI target, adding $0.5 \mu \mathrm{l}$ of a saturated aqueous 2,5-DHB solution, drying the sample as usual.

Sequential solubilization of phosphopeptides using both 2,5-DHB and ammonium phosphate buffers was also carried out, using standard reagents as described above. Briefly, 2,5-DHB treatment was done as above, and the solution was then decanted. Three washes with $20 \mu \mathrm{L}$ water were then carried out before a second attempt at solubilization in $100 \mathrm{mM}$ ammonium phosphate buffer at $\mathrm{pH} 4.8$ was performed. The solutions from both of these treatments were analyzed by MALDI-TOFMS as described earlier.

Finally, we have studied MALDI-TOFMS sensitivity limits for IMAC-enriched phosphopeptides. In these experiments we used a $10 \mu$ l slurry of phosphopeptideloaded $\mathrm{Fe}^{3+}$-NTA beads and the chelated peptides were solubilized with $10 \mu \mathrm{l}$ of ammonium phosphate buffer ( $\mathrm{pH}$ 4.8). MALDI-TOFMS analysis was then performed on $0.5 \mu \mathrm{l}$ of the supernatant $(5 \%)$ with $0.5 \mu \mathrm{l}$ of a saturated aqueous 2,5-DHB solution on a MALDI target using the "dried droplet" preparation technique.

\section{Results}

\section{Direct Analysis}

When phosphopeptide-loaded IMAC beads were applied to the target and mixed with 2,5-DHB matrix solution, we noted that the beads changed color from orange to purple. Upon probing the entire sample spot area on the target by MALDI-TOFMS, prominent ion signals were detected corresponding to mono-phosphorylated peptides as shown in the spectra of Figure 1. Surprisingly, these phosphopeptide signals were obtained only from the crystalline ring surrounding the beads (as would be expected for the normal behavior of an analyte solution using 2,5-DHB as matrix). However, 

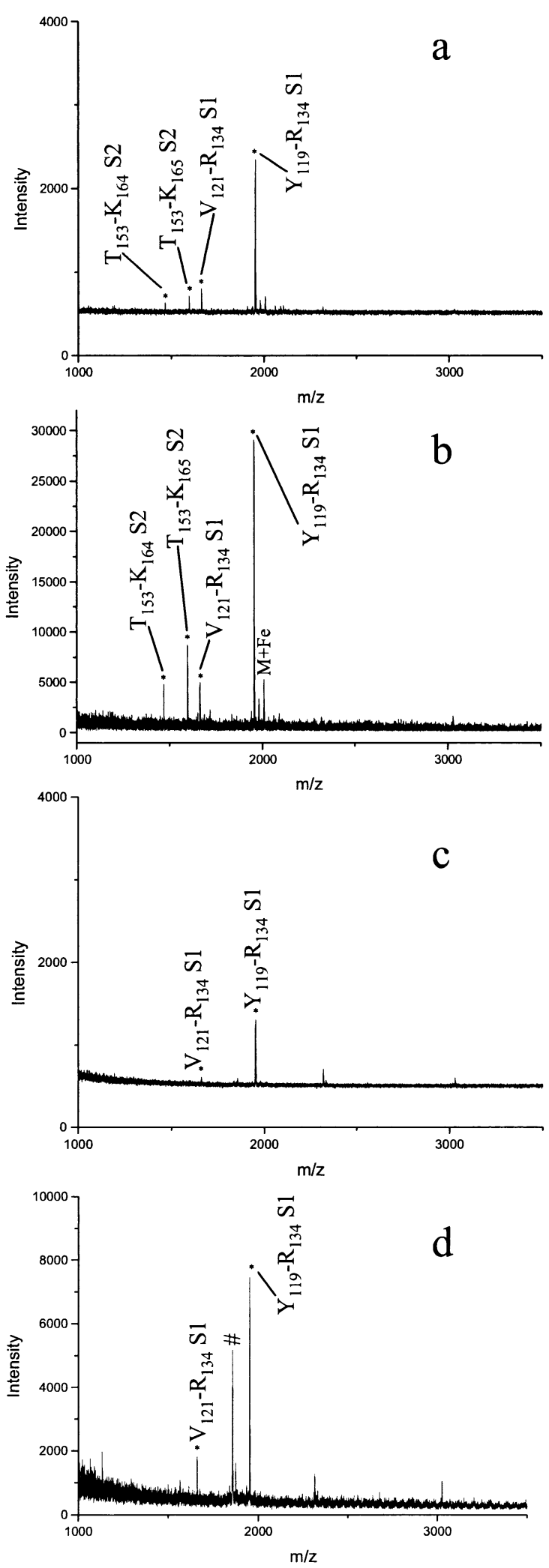

Figure 1. MALDI-TOFMS mass spectra of a tryptic $\alpha$-casein digest acquired after IMAC treatment using the direct analysis method (IMAC beads were loaded directly on the target and matrix was added subsequently). Phosphopeptides are marked by an asterisk. Some non-phosphorylated $\alpha_{\mathrm{S} 1}$-casein peaks are seen $\left(\mathrm{m} / \mathrm{z} 2316.14\left[\mathrm{E}_{148}-\mathrm{R}_{166}\right], 3026.43\left[\mathrm{Y}_{181}-\mathrm{K}_{208}\right]\right)$, cf. Figures 2, 3, 4. These peptides correspond to one tryptic and one non-specifically cleaved peptide, respectively. Their presence can be attributed to affinity-binding of acidic residues and/or putative hydrophobic interaction with $\mathrm{Fe}^{3+}$-NTA IMAC beads, and has frequently been seen in IMAC experiments. Full sequences for labeled phosphopeptides are as follows: $S 1 \mathrm{~V}_{121}-\mathrm{R}_{134}$ : VPQLEIVPNPSAEER, $\mathrm{m} / \mathrm{z}$ 1660.79; $\mathrm{S} 1 \mathrm{Y}_{119}-\mathrm{R}_{134}$ : YKVPQLEIVPNpSAEER, $\mathrm{m} / \mathrm{z}$ 1951.95; S2 $\mathrm{T}_{153}-\mathrm{K}_{164}$ : TVDMEpSTEVFTK, $\mathrm{m} / \mathrm{z} 1466.61$; $2 \mathrm{~T}_{153}-\mathrm{K}_{165}$ : TVDMEpSTEVFTKK, $\mathrm{m} / \mathrm{z}$ 1594.71. (a) 2,5-DHB as matrix in linear mode; (b) 2,5-DHB as matrix in reflectron mode; (c) CHCA as matrix in linear mode; (d) CHCA as matrix in reflectron mode. Prominent metastable decay from the phosphopeptide $Y_{119}-R_{134}$ is marked by a pound sign.

little or no signal for phosphopeptides was observed from the beads themselves. These results suggest that the IMAC bound peptides were being solubilized in the matrix solutions and were thus becoming cocrystallized with matrix as these solutions dry. In order to eliminate any effect of the solvents used for these and further experiments, all solvent systems were tested prior to experimental use. No significant solubilization of phosphopeptides was observed.

In both the linear and reflectron mode, ion signals can be seen representing the tryptic $\alpha_{\mathrm{S} 1}$ casein monophosphopeptides $\mathrm{V}_{121}-\mathrm{R}_{134}$ (VPQLEIVPNPSAEER) and $\mathrm{Y}_{119}-\mathrm{R}_{134}$ (YKVPQLEIVPNPSAEER). However, when 2,5-DHB is used as matrix, two additional mono-phosphorylated $\alpha_{\mathrm{S} 2}$ casein peptide ions $\mathrm{T}_{153}-\mathrm{K}_{164}$ (TVDMEpSTEVFTK) and $\mathrm{T}_{153}-\mathrm{K}_{165}$ (TVDMEpSTEVFTKK) are observed (Figure $1 \mathrm{a}$ and $\mathrm{b}$ ). As anticipated, the use of reflectron mode provides increased mass accuracy and resolution. On the other hand, metastable decay of the phosphopeptide ions occurs as a loss of $\sim 86 \mathrm{Da}$ from the parent ion mass value due to the defocusing of metastable decay fragment ions in reflector mode. As is obvious upon inspection of the spectra shown in Figure 1 , those beads mixed with CHCA matrix solution give comparatively lower peak intensities for the $\alpha$-casein phosphopeptides than 2,5-DHB in both linear and reflectron modes.

Ferric adduct ions were additionally observed when 2,5-DHB was used. These adduct ions were seen with a monoisotopic mass increase of 52.9 Da from the phosphopeptide peak, bearing the characteristic isotope pattern of iron. As judged from the retention of the purple color of the beads, the bulk of the $\mathrm{Fe}^{3+}$ appears to remain bound to the NTA-sepharose beads.

\section{Indirect Analysis}

When 2,5-DHB solution was added to the IMAC-beads in the indirect analysis experiments, the beads immediately changed color from orange to purple just as in the direct analysis experiments. Following centrifugation of this slurry, the supernatant was applied to the target and dried. Both linear and reflectron MALDI-TOFMS spectra were recorded using the 2,5-DHB supernatant (Figure $2 \mathrm{a}$ and $\mathrm{b}$ ). It can be seen that well-defined peaks are present at all of the mono-phosphopeptide mass 

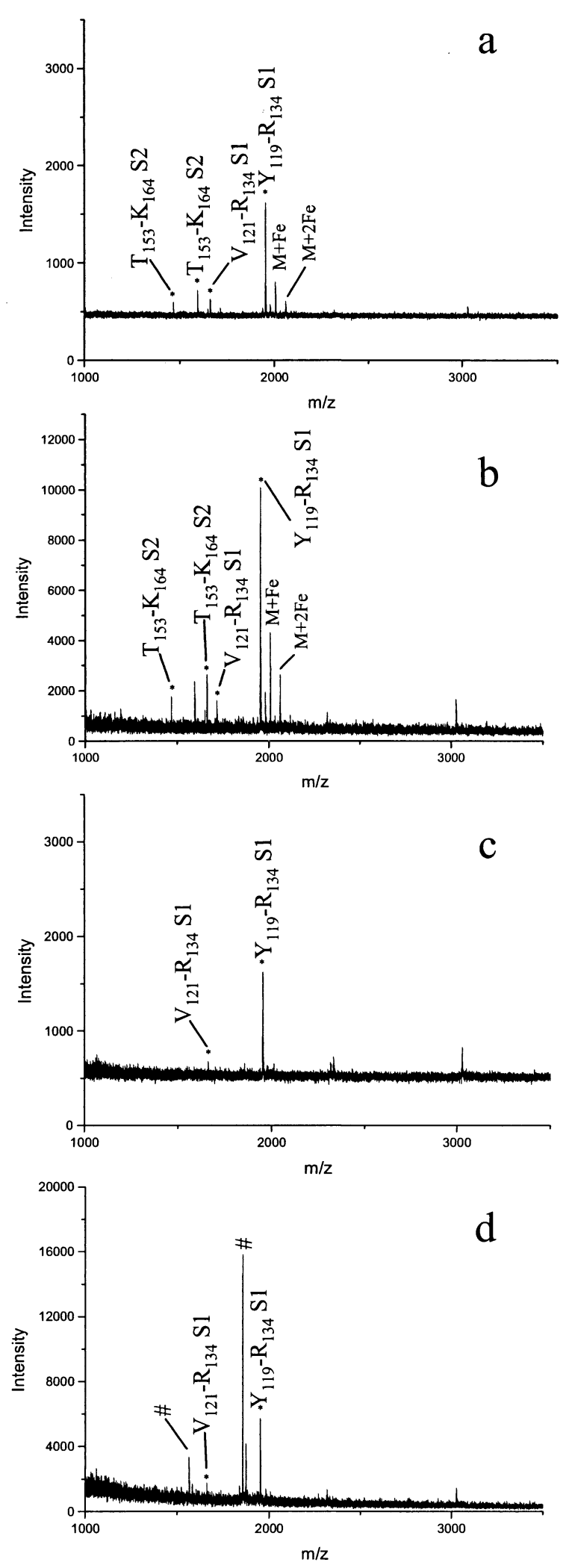

Figure 2. MALDI-TOFMS mass spectra of a tryptic $\alpha$-casein digest acquired after IMAC treatment using the indirect analysis method (IMAC beads were washed with matrix solution and the supernatant was loaded on the target). Phosphopeptides are marked by an asterisk, sequences as in Figure 1. (a) 2,5-DHB as matrix in linear mode; (b) 2,5-DHB as matrix in reflectron mode; (c) CHCA as matrix in linear mode; (d) CHCA as matrix in reflectron mode.
Prominent metastable decay of the phosphopeptides $V_{121}-R_{134}$ and $\mathrm{Y}_{119}-\mathrm{R}_{134}$ is marked by a pound sign.

values observed in Figure 1. This result confirms that the phosphopeptides have in fact been solubilized in the matrix solution as deduced from our first experiments described above.

As with the direct analysis, samples analyzed in 2,5-DHB, when compared to CHCA samples, exhibited higher signal intensities (and thus better signal-to-noise ratios) for the intact $\alpha$-casein phosphopeptide ions, but lower signal intensities for their metastable ions.

Further, ferric $\left(\mathrm{Fe}^{3+}\right)$ peptide adducts were easily observed using 2,5-DHB samples, but were barely detectable using CHCA. Again, the bulk of the $\mathrm{Fe}^{3+}$ appeared to remain bound to the NTA-sepharose beads following treatment with 2,5-DHB, i.e., the beads retained their purple color even after several washes, whereas the supernatant remained clear.

Additional phosphopeptide-solubilization IMAC studies were carried out using the indirect analysis method with different positional isomers of DHB (2,3-, 2,4-, 2,6-, 3,4-, 3,5-DHB and salicylic acid). The spectra derived from these experiments contained fewer phosphopeptide peaks with lower ion signal intensities than the spectra obtained using 2,5-DHB solutions. Addition of 2,5-DHB as MALDI matrix to the positional isomersolubilized fractions did not improve phosphopeptide detection. These results are summarized in Table 1. Table 1 also includes a qualitative description of the color changes of phosphopeptide-loaded IMAC beads upon the addition of different DHB isomer solutions. The color changes were not the same as those obtained with the 2,5 isomer.

\section{pH Variation}

The reflectron mode MALDI-TOFMS spectrum of an untreated tryptic digest is shown in Figure 3a. This spectrum shows that only the $\alpha_{\mathrm{S} 1}$-casein phosphopeptides $\mathrm{Y}_{119}-\mathrm{R}_{134}, \mathrm{~V}_{121}-\mathrm{R}_{134}$, and $\mathrm{Y}_{119}-\mathrm{K}_{139}$ expected from different cleavages of the overall sequence YKVPQLEIVPNpSAEERLHSMK are obvious. The three subsequent panels (Figure 3b, c, d) were obtained using ammonium phosphate buffers of different $\mathrm{pH}$ values covering the range from 4.8-9.6. Surprisingly, very few qualitative differences were evident among these spectra of phosphopeptides, which cover the entire suite of known $\alpha$-casein phosphorylation sites.

\section{Sequential Solubilization (Figure 4)}

In the 2,5-DHB experiments described above, only the mono-phosphorylated components of the phosphopeptide set investigated were detected. Hence, after subjection to the MALDI matrix experiment, the IMAC beads were separated, triply washed with water and extracted 
Table 1. Indirect analysis results using dihydroxybenzoic acid isomers

\begin{tabular}{|c|c|c|c|c|}
\hline Substance structure & $\begin{array}{c}\mathrm{pH} \text { of } \\
\text { saturated } \\
\text { aqueous solution }\end{array}$ & $\begin{array}{c}\text { Colour of } \\
\mathrm{Fe}^{3+}-\mathrm{NTA} \\
\text { beads upon DHB } \\
\text { addition }\end{array}$ & $\begin{array}{c}\text { lon signal } \\
\text { intensities } \\
\text { without } \\
\text { additional } \\
\text { matrix }^{\text {a }}\end{array}$ & $\begin{array}{c}\text { Ion signal } \\
\text { intensities } \\
\text { with } 2,5-\mathrm{DHB} \\
\text { as matrix } \\
\end{array}$ \\
\hline 2,5-DHB & 2.0 & Indigo-purple & Strong & $\mathrm{N} / \mathrm{A}$ \\
\hline $\begin{aligned} 2,3-\mathrm{DHB} \\
\mathrm{O}_{\curlyvee} \mathrm{O}^{-}\end{aligned}$ & 2.4 & Purple & Medium & Medium \\
\hline $\begin{aligned} 2,4-D \\
0\end{aligned}$ & 2.4 & Reddish-purple & None & Medium \\
\hline $\begin{array}{r}2,6-\mathrm{DHB} \\
\mathrm{O}_{\Im} \mathrm{O}\end{array}$ & 1.6 & Reddish-purple & Medium & Medium \\
\hline $\begin{array}{r}3,4-\mathrm{DHB} \\
\mathrm{O}_{Y^{-}}\end{array}$ & 2.8 & Navy blue & None & Weak \\
\hline 3,5-DHB & 2.6 & $\begin{array}{l}\text { Very slight red } \\
\text { colour }\end{array}$ & None & Weak \\
\hline $\begin{array}{l}\text { Salicyclic acid (2-hydroxy } \\
\text { benzoic acid) }\end{array}$ & 2.5 & Slightly red & None & Medium \\
\hline
\end{tabular}

a Indicates the phosphopeptide ion signal intensity when supernatants from the IMAC elution into isomer solution are applied directly to the MALDI target, without addition of additional matrix solution, measured relative to 2,5-DHB (designated as strong).

${ }^{b}$ Indicates the phosphopeptide ion signal intensity when 2,5-DHB is added to the supernatant to assist MALDI.

using conditions found above to disrupt the interaction of all bound phosphopeptides with IMAC resin (elution in ammonium phosphate buffer at $\mathrm{pH} 4.8$ ). The reflectron mode MALDI-TOFMS data obtained from the two treatments (Figure 4) revealed that the poly- and some mono-phosphopeptide had remained bound during treatment with 2,5-DHB solution and the subsequent wash step, but were in fact then released by treatment with the ammonium phosphate buffer. In addition, the interaction between 2,5-DHB and $\mathrm{Fe}^{3+}$-NTA also appeared to be disrupted by the addition of the ammonium phosphate, as suggested by the loss of the purple color of the (DHB-treated) beads.

\section{Sensitivity Study}

In order to determine practical limits for the detection of phosphopeptides from a tryptic digest of bovine $\alpha$-casein, a dilution series was carried out. Results from analysis of $10 \%$ of the eluate (using ammonium phos- phate buffer at $\mathrm{pH} 4.8$ as eluent) with 2,5-DHB as matrix established that all of the major phosphopeptides could be observed using less than 1 pmol total digest loaded. Also, the major phosphopeptides could still be seen at $200 \mathrm{fmol}$ total digest amount loaded, and finally, some highly-abundant phosphopeptides $\left(\alpha_{\mathrm{S} 1} \mathrm{Y}_{119}-\mathrm{R}_{134}, \mathrm{~V}_{121^{-}}\right.$ $\left.\mathrm{R}_{134}, \alpha_{\mathrm{S} 2} \mathrm{~T}_{153}-\mathrm{K}_{165}\right)$ could still be observed at $20 \mathrm{fmol}$ loading levels.

\section{Additional Experiments}

To ensure the quantitative binding of phosphopeptides on IMAC resin HPLC analyses of untreated tryptic $\alpha$-casein digests and the supernatants of the same digests exposed to IMAC beads were carried out followed by MALDI mass analyses of the collected fractions. Comparison of the results obtained from these two HPLC experiments revealed complete depletion of phosphopeptides following exposure to IMAC beads, i.e., UV absorption at $214 \mathrm{~nm}$ was below the detection 

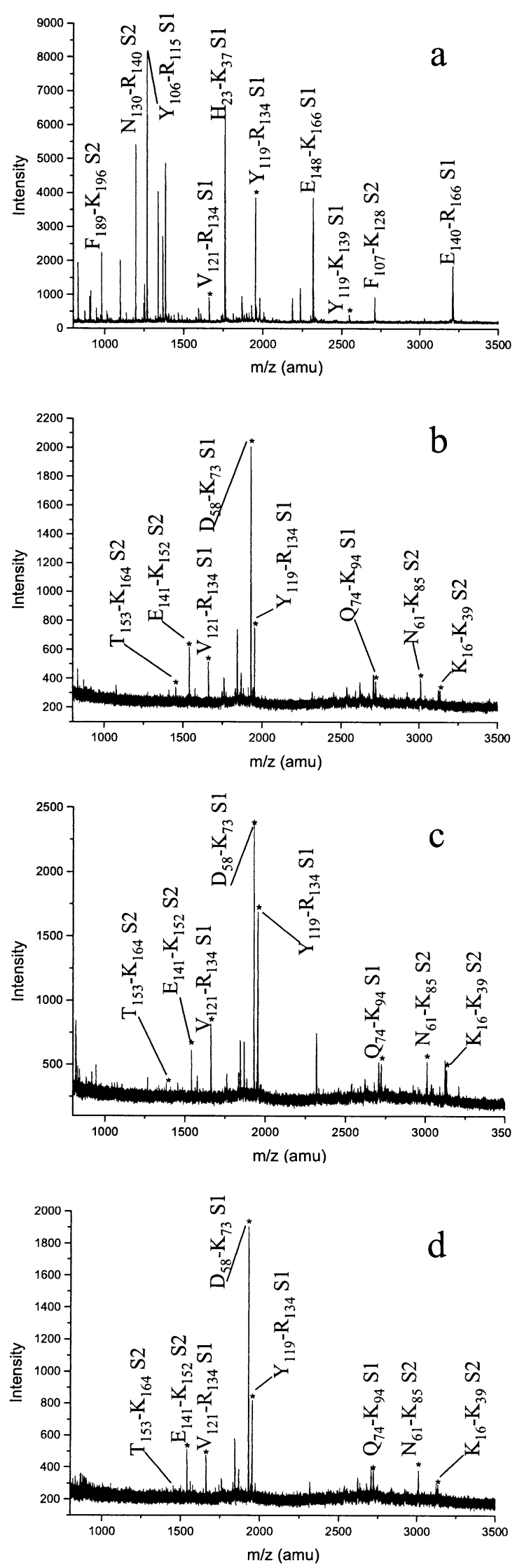

Figure 3. Reflectron MALDI-TOFMS mass spectra of a tryptic $\alpha$-casein digest using 2,5-DHB as matrix. Phosphopeptides are marked by *. Additional phosphopeptides to those in Figures 1 and 2 are present: $\mathrm{S}_{1} \mathrm{D}_{58}-\mathrm{K}_{73}$ : DIGpSEpSTEDQAMEDIK, $\mathrm{m} / \mathrm{z}$ 1927.69; S1 $\mathrm{Q}_{74}-\mathrm{R}_{94}$ QMEAEpSIpSpSpSEEIVPNpSVEQK, $\mathrm{m} / \mathrm{z}$ 2720.91; S2 $\mathrm{E}_{141}-\mathrm{K}_{152}$ EQLPSTpSEENSKK, $\mathrm{m} / \mathrm{z}$ 1539.60; S2 $\mathrm{N}_{61}-\mathrm{K}_{85}$ NANEEEYSIGpSpSpSEEPSAEVATEEVK, $\mathrm{m} / \mathrm{z}$ 3008.03. S2 $\mathrm{K}_{16^{-}}$ $\mathrm{K}_{39}$ KNTMEHVpSpSpSEESIIpSQETYKQEK, m/z 3132.20 (a) Prior to IMAC treatment; (b) After IMAC treatment using ammonium hydroxide/ammonium phosphate elution buffer at $\mathrm{pH} 4.8$; (c) After IMAC treatment using ammonium hydroxide/ammonium phosphate elution buffer at $\mathrm{pH}$ 6.8; (d) After IMAC treatment using ammonium hydroxide/ammonium phosphate elution buffer at $\mathrm{pH}$ 8.6.

threshold level and no phosphopeptide ion signal was observed in any of the fractions by MALDI-MS (data not shown).

\section{Discussion}

Earlier, Tomer and co-workers have reported that direct MALDI could account for the metal-binding peptide signals observed in MALDI-TOFMS spectra obtained by loading IMAC beads onto a MALDI target in CHCA $[9,10]$. These studies were recently extended to the use of $\mathrm{Fe}^{3+}$ - and $\mathrm{Ga}^{3+}$-immobilized beads for the selective detection of phosphopeptides [7]. The data presented in the latter study using $\mathrm{Fe}^{3+}$-modified beads revealed that more highly-phosphorylated peptides could not be detected, even as metastable ions, when CHCA was used as MALDI matrix. This observation is contrary to the data presented by Posewitz and Tempst, who showed that, on average, $\sim 95 \%$ of ${ }^{32}$ P-labelled phosphopeptide was retained by $\mathrm{Fe}^{3+}$ beads under low-pH loading conditions (three separate radiolabelled phosphopeptides were examined) [2]. Since their results showed that radiolabeled phosphopeptides were both present and binding, this result implies that certain additional phosphopeptides were bound to the resin but not detected under these direct MALDI analysis conditions. This discrepancy suggested to us that there must be an alternative property of particular MALDI matrices responsible for these observations. The method of Zhou et al. was therefore revisited in order to determine the physicochemical basis of the direct detection of phosphopeptides chelated to $\mathrm{Fe}^{3+}$-NTA beads.

Our direct analysis experiments show that one can indeed detect mono-phosphopeptides, which have been immobilized to $\mathrm{Fe}^{3+}$-NTA resin, using the methods reported by Tomer et al. Following washing to separate the non-specifically adsorbed components from the specific, immobilized components of the tryptic mixture, the beads were applied directly to the target. Matrix solution was then added to the beads on the target before MALDI-TOFMS analysis. The previous study had shown that CHCA permitted detection of only a limited set of $\alpha$-casein phosphopeptides [7], namely some mono-phosphopeptides. In this study, both CHCA and 2,5-DHB were chosen for investigation 

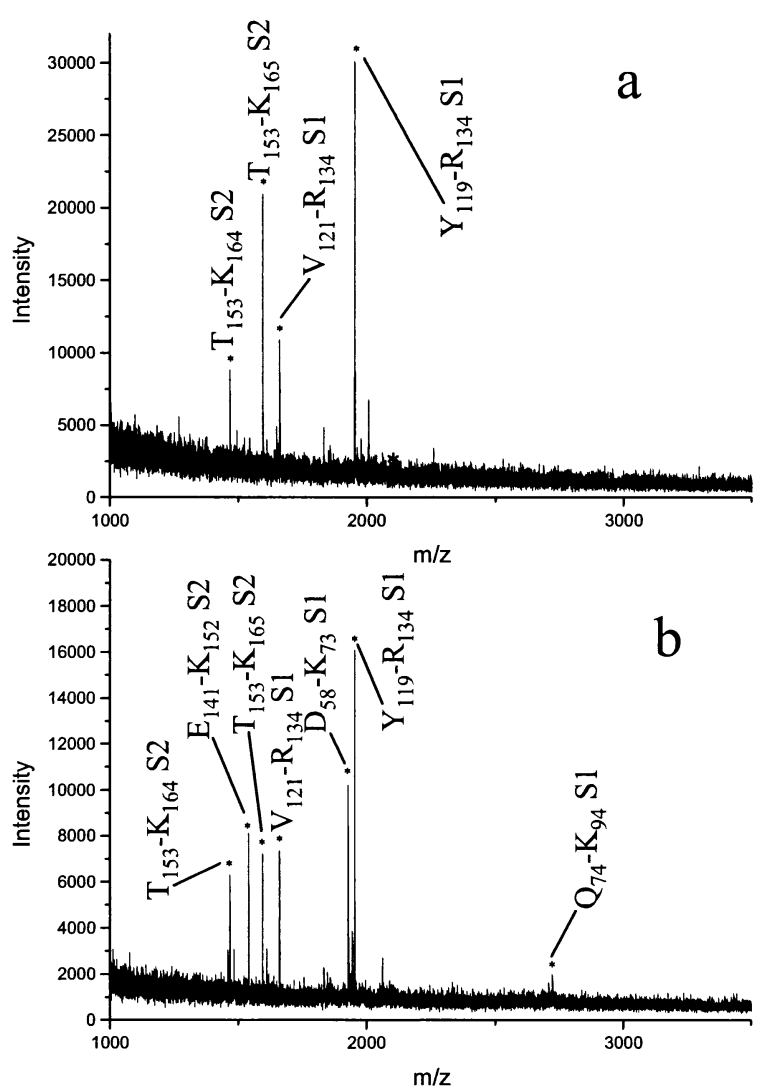

Figure 4. Reflectron MALDI-TOFMS mass spectra of IMACtreated $\alpha$-casein tryptic digest using sequential elution in 2,5-DHB and ammonium phosphate buffer. Phosphopeptides are marked with an asterisk, phosphopeptide masses and sequences are as in the previous figures: (a) IMAC purified fraction eluted using 2,5-DHB, showing mono-phosphopeptide ions. (b) The supernatant from (a) was removed, the beads were washed, and the remaining phosphopeptides eluted using $100 \mathrm{mM}$ ammonium phosphate buffer, $\mathrm{pH} 4.6$.

of the "direct analysis" phenomenon. 2,5-DHB is another MALDI matrix compound, which has been reported to represent a more effective matrix for use in studies of labile phospho- and glycopeptides due to its comparatively "cool" nature (i.e., reduced energy transfer to analyte during the desorption/ionization process) [12-14]. The relatively "hot" nature of CHCA, which under laser desorption conditions causes increased metastable decay of phosphopeptide ions, reduces ion signal intensity for intact phosphopeptide detection, as one might expect. The relative contributions of this limited compatibility of CHCA with phosphopeptide desorption/ionization in comparison with 2,5-DHB, and the differing abilities of these matrices to displace (solubilize) phosphopeptides from the surface of IMAC beads are as yet unclear. It is, however, evident from our studies that samples treated with 2,5-DHB show higher signal intensity for phosphopeptide components. Furthermore, the observation of lower metastable decay with DHB enables the utilization of high-resolution measurements in reflectron mode [15]. However, iron adduct ion formation seems to be significantly higher than when CHCA or ammonium phosphate buffer is employed in elution.

The change in color observed for the IMAC beads treated with 2,5-DHB indicates that there was a chemical interaction between the $\mathrm{Fe}^{3+}$ chelated on the beads and the matrix solution. Phosphopeptide detection from the crystalline ring surrounding the beads suggested that solvation of phosphopeptides had taken place prior to matrix crystallization. One might attribute the phosphopeptide solvation to an effect of the matrix drying process or to their solubilization from the beads into the matrix solution.

Since our initial experiments clearly raised questions about the relative ability of the two matrices examined to solubilize phosphopeptides, we carried out further experiments to investigate the nature of phosphopeptide solubilization from IMAC media using both MALDI matrices and ammonium phosphate buffers with $\mathrm{pH}$ values adjusted throughout the range from acidic to alkaline.

To decide whether phosphopeptide liberation from the beads' surface is taking place by a chemical process, in which the matrix solution can be regarded as the displacing ligand, or by the drying process (as some sort of matrix effect), indirect analysis studies using CHCA and 2,5-DHB were carried out. In these studies, the matrix material was mixed with washed beads, the beads were then spun down and a $0.5 \mu \mathrm{l}$ aliquot of the supernatant was applied to the MALDI target and dried. The spectra gained in the indirect analysis studies (Figure 2) confirmed that MALDI matrix solutions are capable of liberating phosphopeptides, and suggest that the above observations are due to ligand displacement reactions carried out by the matrix rather than a direct desorption effect.

Summarizing the results from the direct and indirect analysis experiments it is shown that the use of 2,5-DHB rather than $\mathrm{CHCA}$ to liberate phosphopeptides from IMAC beads' surfaces allows enhanced detection of ions using reflectron mode. Since only limited metastable fragmentation can be observed with 2,5-DHB, reflectron mode with its increased mass accuracy and resolution can be used to gain more accurate information about the peptide ions present. Phosphopeptide solubilization with 2,5-DHB by either indirect or direct analysis methods also slightly enlarges the phosphopeptide set seen when compared with that seen in CHCA (see Figures 1 and 2). Although the phosphopeptide set found by MALDI-TOFMS after solubilization using MALDI matrices consists only of mono-phosphorylated peptides, two additional phosphopeptides are seen when 2,5-DHB is used instead of CHCA.

That 2,5-DHB and CHCA are in fact able to solubilize mono-phosphopeptides from IMAC beads contradicts the majority of IMAC affinity column elution protocols, which exploit the use of high $\mathrm{pH}$ to disrupt the interaction between immobilized $\mathrm{Fe}^{3+}$ and the bound phosphopeptides $[2,8,16,17]$. The $\mathrm{pH}$ of $2,5-\mathrm{DHB}$ in a saturated aqueous solution is 2.0 , whereas traditional 
IMAC protocols typically use the elution conditions of sodium phosphate at high $\mathrm{pH}(8-10.5)$. From results obtained in this study, solubilization takes place independent of $\mathrm{pH}$, and indicates that liberation of monophosphorylated peptides by 2,5-DHB must be occurring by a chemical ligand displacement mechanism.

Benzoic acid derivatives are known for their high iron affinity, and have been suggested by some groups as bacterial siderophores. Siderophores are low molecular weight chelating agents secreted by microorganisms, which are capable of sequestering $\mathrm{Fe}^{3+}$ for metabolic usage under low $\mathrm{Fe}^{3+}$ conditions $[18,19]$. These results are consistent with the premise that displacement of mono-phosphopeptides by 2,5-DHB may occur as a result of preferential complex formation between the 2,5-DHB molecule and the NTA-complexed $\mathrm{Fe}^{3+}$ over the complex with phosphopeptide.

The indirect analysis experiment carried out using different positional isomers of DHB indicated that although many of the other positional isomers were capable of interacting with the $\mathrm{Fe}^{3+}$-NTA (as indicated by a color change in the resin when the DHB solution was added), the mass spectral data obtained were of substantially lower quality to those where the 2,5isomer was used. It was also noted that the changes in color of the beads, when other isomers were used, were different to the change with 2,5-DHB. Where 2,5-DHB was added to the supernatants from these experiments, the resultant MALDI-TOFMS spectra improved, but still showed fewer phosphopeptide peaks than where 2,5-DHB alone was used. This suggests that the 2,5DHB is better suited for solubilization ("elution") of mono-phosphopeptides immobilized on IMAC beads, although inferior laser desorption/ionization using the other isomers, even when 2,5-DHB is added to improve MALDI analysis, can not be excluded.

Although certain mono-phosphorylated peptides, which are present at very high concentrations in the $\alpha$-casein tryptic digest, were easily seen with $2,5-\mathrm{DHB}$ as matrix solution, the poly-phosphopeptides were difficult to detect. Since HPLC fractionation of the $\alpha$-casein digest before and after IMAC retention shows that these phosphopeptides must be both present and binding to the IMAC media, a lack of phosphopeptide liberation was suspected for certain components. This indicated that a more stringent protocol for the disruption of IMAC bead interaction with hyper-phosphorylated peptides was required, as the strength of interaction between 2,5-DHB and $\mathrm{Fe}^{3+}$-NTA appears to be insufficient to disrupt the interaction of multiply phosphorylated peptides with IMAC resin. Since in nature phosphorylation sites have a propensity to occur in clusters, this is a critical consideration which needs to be addressed. A generic method for solubilization of chelated peptides from $\mathrm{Fe}^{3+}$-NTA beads was therefore sought.

Obviously, compatibility with mass spectrometric analysis without downstream purification of IMACenriched phosphopeptide samples was desired. The use of sodium salt solutions was therefore somewhat unde- sirable, although widely used in IMAC [2, 20-22]. The method reported by Gallis et al. [3] involves the use of monobasic ammonium phosphate $\left(\mathrm{NH}_{4} \mathrm{H}_{2} \mathrm{PO}_{4}\right)$ in a $0.1 \%$ aqueous solution; a method analogous to that used by other groups with sodium phosphate. Ammonium phosphate solution not only proved to be MALDITOFMS compatible, but also enabled detection of multiply phosphorylated peptides, and can be $\mathrm{pH}$-buffered. Therefore, $\mathrm{pH}$-adjusted ammonium phosphate buffers were used to investigate whether $\mathrm{pH}$ affects phosphopeptide recovery from IMAC beads. $\mathrm{pH}$-dependent elution of phosphopeptides from IMAC resin is often a fundamental aspect of literature protocols, whose necessity was brought into question by our results showing mono-phosphopeptide solubilization using MALDI matrix solutions. MALDI matrices are acidic; chelate disruption in these solution conditions is therefore contrary to the disruption conditions employed in standard column elution protocols. Further evidence for the lack of necessity for high $\mathrm{pH}$ elution was found in some of the earliest protocols, where elution was carried out at $\mathrm{pH} 7.2$ [1]. The data from three independent experiments carried out over a range of $\mathrm{pH}$ values revealed no significant compositional change due to the difference in $\mathrm{pH}$. Figure 3 depicts typical spectra obtained for the $\mathrm{pH}$ values $4.8,6.8$, and 8.6. These results indicate that the usually very low $\mathrm{pH}$ of MALDI matrices imposes no essential disadvantage when it is used with IMAC eluents. On the contrary, the high $\mathrm{pH}$ of some column elution buffers could cause problems such as $\beta$-elimination of phosphate from phosphoserine and phosphothreonine residues, which is known to take place at high $\mathrm{pH}[23]$.

Since we have shown in this study that ammonium phosphate disrupts the interaction of multiply phosphorylated peptides where 2,5-DHB could not, initial experiments to determine the physical properties of 2,5-DHB's solubilization capacity were carried out. Sequential elution with 2,5-DHB, followed by ammonium phosphate, showed that the multiply phosphorylated and even some mono-phosphorylated peptides were still bound to the resin and could be solubilized using an ammonium phosphate buffer subsequent to treatment with 2,5-DHB (Figure 4). The purple color of the beads was lost following treatment with ammonium phosphate, indicating that the affinity of phosphate ions for the resin is, as would be expected, higher than that of 2,5-DHB or CHCA. This explains the lack of multiply-phosphorylated peptide ions when these matrices are applied to $\mathrm{Fe}^{3+}$-IMAC beads and directly analyzed on target $[7,9]$.

The recovery of the complete set of phosphopeptides gained from tryptic digestion, and therefore characterization of the phosphorylation profile of this protein, is therefore achievable using either this combination of 2,5-DHB and ammonium phosphate or just ammonium phosphate alone. 


\section{Conclusion}

The results obtained in this study provide strong evidence that the "direct analysis" phenomenon in MALDI-TOFMS analysis of IMAC-retained phosphopeptides is in fact due to ligand displacement of the mono-phosphopeptide-NTA chelate, rather than laserinduced direct desorption from the chelate. IMACretained phosphopeptides can be solubilized by solutions of CHCA and $\mathrm{DHB}$, with 2,5-DHB being more effective in obtaining a larger subset of mono-phosphopeptides.

For the liberation of multiply phosphorylated peptides, buffers containing phosphate were superior to 2,5-DHB or other MALDI matrix solutions. Elution from IMAC beads under these conditions is related to increasing phosphate concentration at the beads' surface rather than $\mathrm{pH}$, since this study finds no significant alterations in the elution profile in the $\mathrm{pH}$ range from 4.8-9.6. Ammonium phosphate does not strongly interfere with MALDI or HPLC measurements of phosphopeptides, its $\mathrm{pH}$ can be adjusted to maintain phosphopeptides in solution, and most importantly, it efficiently solubilizes mono- as well as poly-phosphopeptides from $\mathrm{Fe}^{3+}$-NTA beads. This would therefore appear to be an optimal choice as an IMAC elution buffer.

In summary, the findings of this study indicate that the metal chelation aspects of IMAC methods for phosphopeptide enrichment need to be considered much more carefully than previous studies would imply. The ability of $\mathrm{Fe}^{3+}$-chelating agents, such as 2,5-DHB, to displace phosphopeptide from IMAC media at low $\mathrm{pH}$ is a first indication that differential chelation complexes at the metal ions' surface are more important than $\mathrm{pH}$ changes for displacement of these components. Further research into the chelation aspects of IMAC separation is therefore clearly desirable.

\section{Acknowledgments}

SRH is supported by the Medical Research Council. Support for instrumentation was provided by the LICR.

\section{References}

1. Andersson, L.; Porath, J. Isolation of Phosphoproteins by Immobilized Metal $(\mathrm{Fe} 3+)$ Affinity Chromatography. Anal. Biochem. 1986, 154, 250-254.

2. Posewitz, M. C.; Tempst, P. Immobilized Gallium(III) Affinity Chromatography of Phosphopeptides. Anal. Chem. 1999, 71, 2883-2892.

3. Gallis, B.; Corthals, G. L.; Goodlett, D. R.; Ueba, H.; Kim, F.; Presnell, S. R.; Figeys, D.; Harrison, D. G.; Berk, B. C.; Aebersold, R.; Corson, M. A. Identification of Flow-Dependent Endothelial Nitric-Oxide Synthase Phosphorylation Sites by Mass Spectrometry and Regulation of Phosphorylation and Nitric Oxide Production by the Phosphatidylinositol 3-Kinase Inhibitor LY294002. J. Biol. Chem. 1999, 274, 30101-30108.

4. Li, S. H.; Dass, C. Iron(lll)-Immobilized Metal Ion Affinity Chromatography and Mass Spectrometry for the Purification and Characterization of Synthetic Phosphopeptides. Anal. Biochem. 1999, 270, 9-14.

5. Stensballe, A.; Andersen, S.; Jensen, O. N. Characterization of Phosphoproteins from Electrophoretic Gels by Nanoscale $\mathrm{Fe}(\mathrm{III})$ Affinity Chromatography with Off-Line Mass Spectrometry Analysis. Proteomics 2001, 1, 207-222.

6. Chaga, G. S. Twenty-Five Years of Immobilized Metal Ion Affinity Chromatography: Past, Present, and Future. J. Biochem. Biophys. Methods 2001, 49, 313-334.

7. Zhou, W.; Merrick, B. A.; Khaledi, M. G.; Tomer, K. B. Detection and Sequencing of Phosphopeptides Affinity Bound to Immobilized Metal Ion Beads by Matrix-Assisted Laser Desorption/Ionization Mass Spectrometry. J. Am. Soc. Mass Spectrom. 2000, 11, 273-282.

8. Nuwaysir, L. M.; Stults, J. T. Electrospray-Ionization MassSpectrometry of Phosphopeptides Isolated by Online Immobilized Metal-Ion Affinity-Chromatography. J. Am. Soc. Mass Spectrom. 1993, 4, 662-669.

9. Papac, D. I.; Hoyes, J.; Tomer, K. B. Direct Analysis of Affinity-Bound Analytes by Maldi/TOF MS. Anal. Chem. 1994, 66, 2609-2613.

10. Qian, X. H.; Zhou, W.; Khaledi, M. G.; Tomer, K. B. Direct Analysis of the Products of Sequential Cleavages of Peptides and Proteins Affinity-Bound to Immobilized Metal Ion Beads by Matrix-Assisted Laser Desorption/Ionization Mass Spectrometry. Anal. Biochem. 1999, 274, 174-180.

11. Hochuli, E.; Dobeli, H.; Schacher, A. New Metal Chelate Adsorbent Selective for Proteins and Peptides Containing Neighbouring Histidine Residues. J. Chromatogr. 1987, 411, 177-184.

12. Cramer, R.; Richter, W. J.; Stimson, E.; Burlingame, A. L. Analysis of Phospho- and Glycopolypeptides with Infrared Matrix-Assisted Laser Desorption and Ionization. Anal. Chem. 1998, 70, 4939-4944.

13. Cramer, R.; Burlingame, A. L. IR-MALDI-Softer Ionization in MALDI-MS for Studies of Labile Macromolecules. In Mass Spectrometry in Biology and Medicine; Burlingame, A. L.; Carr, S. A.; Baldwin, M. A., Eds.; Humana Press: Totowa, New Jersey, 2000; pp 289-307.

14. Stimson, E.; Truong, O.; Richter, W.; Waterfield, M.; Burlingame, A. L. Enhancement of Charge Remote Fragmentation in Protonated Peptides by High-Energy CID MALDI-TOF-MS Using “Cold" Matrices. Int. J. Mass Spectrom. Ion Processes 1997, 169/170, 231-240.

15. Annan, R. S.; Carr, S. A. Phosphopeptide Analysis by MatrixAssisted Laser Desorption Time-of-Flight Mass Spectrometry. Anal. Chem. 1996, 68, 3413-3421.

16. Anguenot, R.; Yelle, S.; Nguyen-Quoc, B. Purification of Tomato Sucrose Synthase Phosphorylated Isoforms by Fe(III)Immobilized Metal Affinity Chromatography. Arch. Biochem. Biophys. 1999, 365, 163-169.

17. Neville, D. C.; Rozanas, C. R.; Tulk, B. M.; Townsend, R. R.; Verkman, A. S. Expression and Characterization of the NBD1-R Domain Region of CFTR: Evidence for SubunitSubunit Interactions. Biochemistry 1998, 37, 2401-2409.

18. Carrano, C. J.; Drechsel, H.; Kaiser, D.; Jung, G.; Matzanke, B.; Winkelmann, G.; Rochel, N.; Albrecht-Gary, A. M. Coordination Chemistry of the Carboxylate Type Siderophore Rhizoferrin: The iron(III) Complex and Its Metal Analogs. Inorg. Chem. 1996, 35, 6429-6436.

19. Lopez-Goni, I.; Moriyon, I.; Neilands, J. B. Identification of 2,3-Dihydroxybenzoic Acid as a Brucella Abortus Siderophore. Infect. Immun. 1992, 60, 4496-4503.

20. Lin, F.-Y.; Chen, W.-Y.; Huang, S.-Y. Selective Separation of Caseinophosphopeptides Through Immobilized Metal Ion Af- 
finity Chromatography: Effects of $\mathrm{pH}$ Values, Salt Concentrations and Degree of Phosphorylation. Bioproc. Eng. 2000, 23, 467-471.

21. Scanff, P.; Yvon, M.; Pelissier, J. P. Immobilized Fe3+ Affinity Chromatographic Isolation of Phosphopeptides. J. Chromatogr. 1991, 539, 425-432.

22. Zarling, A. L.; Ficarro, S. B.; White, F. M.; Shabanowitz, J.; Hunt, D. F.; Engelhard, V. H. Phosphorylated Peptides are
Naturally Processed and Presented by Major Histocompatibility Complex Class I Molecules in Vivo. J. Exp. Med. 2000, 192, $1755-1762$.

23. Jaffe, H.; Veeranna; Pant, H. C. Characterization of Serine and Threonine Phosphorylation Sites in $\beta$-Elimination/Ethanethiol Addition-Modified Proteins by Electrospray Tandem Mass Spectrometry and Database Searching. Biochemistry 1998, 37, 16211-16224. 